\title{
Research Paper: Inter-rater Reliability, Test-retest Reliability, and Internal Consistency of the Persian Version of Dynamic Gait Index in Patients With Multiple Sclerosis
}

\author{
Hossein Soltanpour ${ }^{1}$ (D), Minoo Kalantari ${ }^{1}$ (D), Meysam Roostaei ${ }^{{ }^{*}}$ (D)
}

1. Department of Occupational Therapy, School of Rehabilitation, Shahid Beheshti University of Medical Sciences, Tehran, Iran.

2. Department of Occupational Therapy, School of Rehabilitation, Tehran University of Medical Sciences, Tehran, Iran.

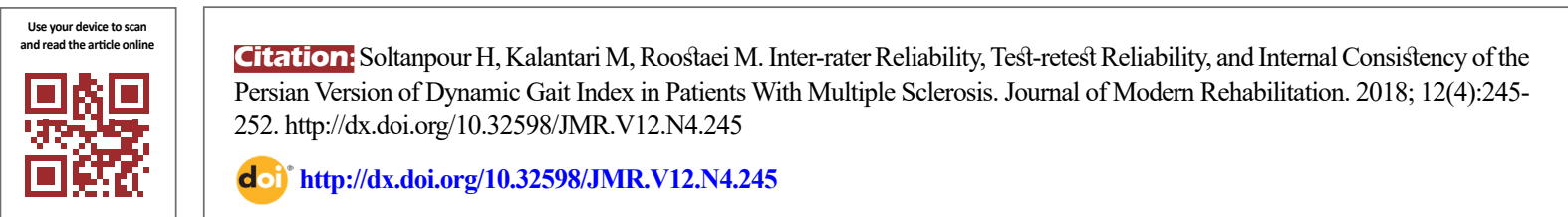

Article info:

Received: 24 Apr 2018

Accepted: 08 Aug 2018

Available Online: 01 Oct 2018

Keywords:

Multiple sclerosis,

Gait, Postural balance,

Psychometrics

\begin{abstract}
A B S T RACT
Introduction: Dynamic Gait Index (DGI) has been used as a valid and reliable tool to assess balance performance in various populations. However, it has been translated into the Persian language and has shown perfect psychometric properties in the elderly population. The Persian version in Multiple Sclerosis (MS) population was validated, but its reliability has not been examined. The aim of the current study was to investigate the inter-rater reliability, test-retest reliability, and internal consistency of the Persian version of DGI in MS patients.
\end{abstract}

Materials and Methods: A total of 62 participants took part in the reliability process. The sample included 51 females and 11 males. Inter-rater reliability was assessed by the agreement of the results obtained by two occupational therapists. We considered a 3-day interval for the first and second data collection. Test-retest and inter-rater reliability were measured with the Intraclass Correlation Coefficient (ICC2, 1) and internal consistency was evaluated with the Cronbach alpha.

Results: The Persian version of DGI showed excellent inter-rater reliability (ICC for total score was 0.98 and ICC for the items ranged from 0.83 to 0.96 ) and test-retest reliability (ICC for the overall score was 0.97 and ICC for the items ranged from 0.82 to 0.96 ). The Cronbach alpha of the Persian version of DGI was 0.87 .

Conclusion: The Persian version of DGI can be used as a reliable tool for dynamic balance measurement of MS patients in clinical settings and research studies. 


\section{Introduction}

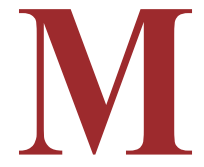

ultiple Sclerosis (MS) is a neurodegenerative disease as a result of demyelination in anywhere of the Central Nerve System (CNS), creating several neurological impairments such as paresthesia, spasticity, numbness, poor motor control, fatigue, difficulty walking, cognitive problems, bowel and bladder problems, vision problems, dizziness or vertigo, pain, and balance deficits $[1,2]$. Balance dysfunction in dynamic and static activities, as well as walking performance, is common among people with MS. Several cross-sectional studies show that the participants with any type or severity of MS indicated significant static and dynamic balance dysfunction compared with typical age-matched control groups $[3,4]$.

A systematic review and meta-analysis by Comber et al. reviewed gait parameters in the people with MS, and their results showed that MS has a significant effect on stride length, step length, cadence, velocity, and swing phase duration. [5]. In spite of applying compensatory strategies, these impairments may increase the rate of falling, fear of falling, low quality of life, and dependency in the activity of daily living [6-9].

Previous studies suggested that the involvement of the infratentorial area, insufficient connectivity of corticocerebellar circuit, cerebellar and brainstem impairment, and lower gray matter volume damage the balance of MS patients [10-13]. Therefore, balance control may become more dependent on well-functioning in cerebellar-regulated proprioceptive controls system, the vestibular system, and the visual system [14]. In addition, Jones demonstrated that one of the essential aspects of establishing balance control is the somatosensory system [15]. However, brain damage can lead to various difficulties in functional tasks that occur as a result of balance dysfunction.

Physical evaluation is the most crucial component in clinical reasoning. Physical and occupational therapists and other practitioners are looking for valid and reliable tools for assessment in the clinical setting. A comprehensive evaluation of balance may lead to a clear understanding of walking performance and assist the therapists in developing therapeutic goals and intervention planning [3]. Dynamic Gait Index (DGI) may be a more proper functional test for people with MS because of easy implementation, and that is a cost-effective measurement.
DGI was introduced in 1995 by Shumway-Cook et al. $[16,17]$. It has 8 items and was designed to evaluate gait quality and balance dysfunction during a walking performance. It has been widely used in several neurological diseases and conditions such as stroke, vestibular disorder, brain injury, and elderly [18-20]. DGI is a valid and reliable test for the MS population [21, 22]. It has been translated into the Persian language and has shown perfect psychometric properties in the elderly population [23]. Also, the Persian version in MS population was validated, but its reliability has not been examined. [24]. Therefore, the current study aimed to investigate the inter-rater reliability, test-retest reliability, and internal consistency of the Persian version of DGI in MS patients.

\section{Materials and Methods}

Guidelines for Reporting Reliability and Agreement Studies (GRRAS) have 15 helpful instructions for reporting a reliability study [25]. We used GRRAS method for reporting all parts of the article.

\section{Study participants}

The participants were selected from Iranian MS Society (IMSS), Imam Hossein Hospital, and Sina Hospital, using the convenience sampling method. The inclusion criteria for MS patients were: 1. Being 20 years or older; 2 . Having disability between 2.0 and 6.0 based on the Expanded Disability Status Scores (EDSS); 3. Being in remission period of MS and consistency in medication; 4. Experiencing balance dysfunction and gait deviation; 5. Being able to walk $10 \mathrm{~m}$ or more and 6 . Having a score of MiniMental Status Examination (MMSE) higher than 22. The exclusion criteria consisted of having one or more comorbid diseases and insufficient collaboration during the test completion.

\section{Study procedure}

Initially, all participants were examined by a neurologist, and after diagnosis, he assigned the EDSS score to the participants. An occupational therapist evaluated MMSE score and considered the inclusion and exclusion criteria. At the first session, the patients completed a demographic questionnaire; also, the occupational therapist exclusively observed all participations during performing the Persian version of DGI items and graded their function according to manual instructions. The retest for the Persian version of DGI was fulfilled three days after the first session. Also, inter-rater reliability was investigated by the agreement of the results obtained 
by the first and second occupational therapists for the total scores of the Persian version of DGI.

All therapists had at least two years of clinical experience in the field of neurological rehabilitation. Observations were made simultaneously by the therapists in the same situation (the same weather, time of year, time of day, and environment), and they were blinded to their scoring process

Dynamic gait index

DGI includes eight items, and each item has a fourpoint rating scale (zero to three). All items are related to the balance function during gait performance. Items consist of gait level surface, change in gait speed (fast to slow), gait with horizontal head turns, gait with vertical head turns, gait and pivot turn, step over the obstacle, step around obstacles, and steps. This survey takes ten minutes or less to be completed [26]. The items are scored on a 4-point scale according to the therapist's evaluation of the participant's performance in each item, where $0=$ Extremely deficit, $1=$ Medium deficit, $2=$ Mild deficit, and 3= Typical performance. The total score of the DGI range from 0-24. A higher score shows a better degree of balance competency and scores less than 19 are defined as a fall risk in MS population [22].

\section{Statistical analysis}

The data were analyzed using SPSS V. 20. Descriptive statistics present The characteristics of the participants and demographic information (age, gender, married situation, etc.) Test-retest and inter-rater reliability were determined by calculating two-way random effects of the Intraclass Correlation Coefficient (ICC2, 1) because DGI was filled out by the same therapists. ICC $<0.4$ is interpreted as a poor agreement; ICC $\geq 0.75$ is excellent agreement, and ICC between these ranges is identified moderate to good [27]. The Cronbach alpha was used for calculating the internal consistency. If the Cronbach alpha is $>0.80$, the internal consistency will be excellent. If it is between 0.70 and 0.80 , the internal consistency will be adequate, and if it is $<0.70$, the internal consistency will be low or inadequate [27].

\section{Results}

A total of 62 patients with MS participated in this study; 41(66\%) were recruited from IMSS, and 21(34\%) were in outpatient wards of Imam Hossein and Sina hospitals. The sample included 51(82\%) females and $11(18 \%)$ males. The Mean \pm SD age of the participants was 38.32 \pm 11.32 , ranging from 20-63; their Mean \pm SD EDSS score was $3.43 \pm 1.34$ and Mean \pm SD MMSE score was $27.82 \pm 2.31$. Table 1 presents the descriptions of the participants.

\section{Inter-rater reliability}

The agreement between the two therapists was checked. The Mean \pm SD score of DGI was $19.13 \pm 3.63$ for the first therapist and $19 \pm 3.70$ for the second therapist. The inter-rater reliability in the total score of DGI was $0.98(95 \%$ confidence interval), and Table 2 presents the result of ICC for each item. All items had excellent reliability.

\section{Test-retest reliability}

The retest data collection session was performed on the same occasion; 26 patients did not participate in the retest procedure because they did not devote their time to participate in the retest session. ICC for the total score of DGI was 0.97 (95\% confidence interval). Table 3 presents ICC for the item scores. ICC for all items shows an excellent agreement.

\section{Internal consistency}

The homogeneity of the items was evaluated by internal consistency. Finding are demonstrated in Table 4. Total Cronbach alpha value was 0.87 . Therefore, the result shows that all items have excellent consistency and measure similar objectives.

\section{Discussion}

The evaluation of dynamic balance in fundamental motor skills like walking is the essential component related to the rehabilitation services. The assessment of balance with a reliable tool is critical for Iranian patients with MS. The present study aimed to evaluate the interrater reliability, test-retest reliability, and internal consistency of the Persian version of DGI in patients with MS. The finding of the present study indicated that the Persian version of DGI showed excellent inter-rater reliability, test-retest reliability, and internal consistency when assessed in a mild to moderate disabled sample of patients with MS.

The agreement between different assessors is in accordance with the findings reported by McConvey et al. [21]. They investigated inter-rater reliability for DGI scores in patients with MS. Inter-rater reliability was conducted by 11 physical therapists, and they used re- 
Table 1. Descriptive characteristics $(n=62)$

\begin{tabular}{|c|c|c|}
\hline Variables & & No. (\%) \\
\hline \multirow{3}{*}{ Gender } & Male & $11(17.7)$ \\
\hline & & \\
\hline & Female & $51(82.3)$ \\
\hline \multirow{3}{*}{ Type of MS } & Relapsing-remitting & $48(77.4)$ \\
\hline & Secondary progressive & $11(17.7)$ \\
\hline & Primary progressive & $3(4.8)$ \\
\hline \multirow{3}{*}{ Married situation } & Married & $48(77.4)$ \\
\hline & & \\
\hline & Single & 14(22.6) \\
\hline \multirow{7}{*}{ The initial symptom of the disease } & Visual impairments & 25(40.3) \\
\hline & Sensory impairments & 19(30.6) \\
\hline & Weakness & $7(11.3)$ \\
\hline & & \\
\hline & Balance dysfunction & 1(1.6) \\
\hline & Dizziness & $1(1.6)$ \\
\hline & Several symptoms & $9(14.5)$ \\
\hline \multirow{3}{*}{ Usage of outpatient rehabilitation programs } & Yes & $18(29)$ \\
\hline & & \\
\hline & No & $44(71)$ \\
\hline
\end{tabular}

cording videotape of participant's performance for reliability process. The results of this study indicated that ICC for total scores in intra-rater reliability of DGI was 0.98 .
Also, their finding showed that ICC for items ranged from 0.91-0.97. These results are similar to another study conducted by Cattaneo et al. [28]; their results demon-

Table 2. Item ICC for inter-rater reliability $(n=62)$

\begin{tabular}{|c|c|c|c|}
\hline \multirow{2}{*}{ Items } & \multirow{2}{*}{ ICC } & \multicolumn{2}{|c|}{ 95\% Confidence Interval } \\
\hline & & Lower Bound & Upper Bound \\
\hline Gait level surface & 0.84 & 0.74 & 0.90 \\
\hline Change in gait speed & 0.93 & 0.88 & 0.95 \\
\hline Gait with horizontal head turns & 0.92 & 0.87 & 0.95 \\
\hline Gait with vertical head turns & 0.83 & 0.73 & 0.89 \\
\hline Gait and pivot turn & 0.94 & 0.90 & 0.96 \\
\hline Step over obstacle & 0.96 & 0.94 & 0.97 \\
\hline Step around obstacles & 0.96 & 0.94 & 0.98 \\
\hline Steps & 0.93 & 0.89 & 0.96 \\
\hline Total & 0.98 & 0.97 & 0.99 \\
\hline
\end{tabular}


Table 3. Item ICC for test-retest reliability $(n=36)$

\begin{tabular}{|c|c|c|c|}
\hline \multirow{2}{*}{ Items } & \multirow{2}{*}{ ICC } & \multicolumn{2}{|c|}{ 95\% Confidence Interval } \\
\hline & & Lower Bound & Upper Bound \\
\hline Gait level surface & 1 & 1 & 1 \\
\hline Change in gait speed & 0.94 & 0.89 & 0.97 \\
\hline Gait with horizontal head turns & 0.94 & 0.89 & 0.97 \\
\hline Gait with vertical head turns & 0.92 & 0.85 & 0.96 \\
\hline Gait and pivot turn & 0.82 & 0.85 & 0.96 \\
\hline Step over obstacle & 0.95 & 0.91 & 0.97 \\
\hline Step around obstacles & 0.96 & 0.93 & 0.98 \\
\hline Steps & 0.95 & 0.92 & 0.97 \\
\hline Total & 0.97 & 0.94 & 0.98 \\
\hline
\end{tabular}

$J \mathrm{MR}$

strated that ICC in intra-rater reliability was 0.94 and expressed that DGI was a reliable tool for patients with MS.

Test-retest reliability is a valuable factor that indicates the stability of result at over time. We considered a threeday interval without rehabilitation and pharmacological interventions for the first and second data collection. The result of this study shows that ICC for test-retest reliability of the Persian version of DGI in patients with MS is 0.97 . This finding was 0.85 and $0.76-0.98$ (for 11 physical therapist) in Cattaneo et al. and McConvey et al.'s studies, respectively $[21,28]$.

Table 4. Cronbach alpha for the eight items in DGI

\begin{tabular}{|c|c|c|c|c|c|c|c|c|c|c|c|}
\hline \multirow[b]{2}{*}{ Items } & \multicolumn{8}{|c|}{ Inter Item Correlation Matrix } & \multirow{2}{*}{ 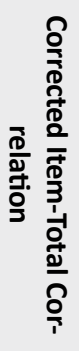 } & \multirow[b]{2}{*}{ 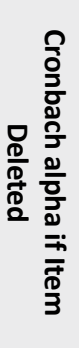 } & \multirow[b]{2}{*}{ 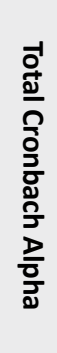 } \\
\hline & 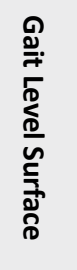 & 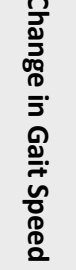 & 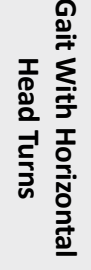 & 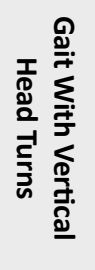 & 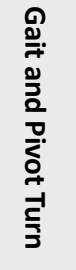 & $\begin{array}{l}\frac{n}{0} \\
\frac{0}{0} \\
0 \\
\frac{0}{1} \\
\frac{1}{0} \\
\frac{0}{0} \\
\frac{0}{D} \\
\frac{0}{D}\end{array}$ & 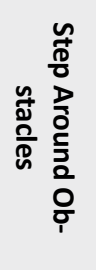 & 竞 & & & \\
\hline Gait level surface & 1.00 & & & & & & & & 0.68 & 0.85 & \\
\hline Change in gait speed & 0.53 & 1.00 & & & & & & & 0.64 & 0.85 & \\
\hline $\begin{array}{c}\text { Gait with horizontal head } \\
\text { turns }\end{array}$ & 0.59 & 0.49 & 1.00 & & & & & & 0.71 & 0.84 & \\
\hline $\begin{array}{l}\text { Gait with vertical head } \\
\text { turns }\end{array}$ & 0.56 & 0.49 & 0.70 & 1.00 & & & & & 0.68 & 0.85 & 0.87 \\
\hline Gait and pivot turn & 0.56 & 0.57 & 0.58 & 0.51 & 1.00 & & & & 0.75 & 0.84 & \\
\hline Step over obstacle & 0.43 & 0.46 & 0.46 & 0.43 & 0.53 & 1.00 & & & 0.58 & 0.86 & \\
\hline Step around obstacles & 0.51 & 0.45 & 0.56 & 0.50 & 0.65 & 0.42 & 1.00 & & 0.65 & 0.85 & \\
\hline Steps & 0.29 & 0.38 & 0.25 & 0.31 & 0.43 & 0.30 & 0.26 & 1.00 & 0.41 & 0.87 & \\
\hline
\end{tabular}


Therefore, our finding is concurrent with the previous studies, and this tool has an acceptable test-retest reliability for patients with MS. Similar to inter-rater and test-retest reliability, the internal consistency was excellent. This result demonstrates that the items of the Persian version of DGI are homogeneous.

Some issues might affect the reliability of the procedure and results. Firstly, the progress of symptoms and fatigue experience can be influential factors in gait performance and defined as a problematic issue in patients with MS diagnosis [29]. Videotape recording is a helpful approach for resolving this challenge and control of disturbances factors in the reliability of the procedure [30]. We did not utilize videotape for the rating process; however, McConvey et al. used videotaping method, and our findings have supported their results. Secondly, the use of multiple expert therapists can lead to stronger reliability outcomes. In this study, only two assessors performed the evaluation in the inter-rater reliability.

Thirdly, all participants had a mild to moderate disability $(\mathrm{EDSS}=3.43 \pm 1.34$ and $\mathrm{MMSE}=27.82 \pm 2.31)$; therefore, the Persian version of DGI may be less reliable in individuals with severe cognitive or motor impairments and the results of this study cannot be generalized to other levels of disability. Fourthly, the type of MS should have been considered as an essential contributing factor in balance dysfunction. Patients with secondary progressive MS have worse balance impairment than relapsing-remitting and primary progressive MS [31]. In this regard, the findings of this study may have insufficient accuracy for the different types of MS.

The Persian version of DGI shows excellent reliability for total and items scores. It can be used as a reliable tool for dynamic balance measurement of MS patients in clinical settings and research studies. Additional studies are required to investigate the validity and reliability of the Persian version of DGI in other populations.

A reliable, functional test is used by therapists in clinical settings to represent the clients' risk of falling events. The total scores of the Persian version of DGI are used to identify individuals who are at risk of falling, and to investigate the dynamic balance change among community-dwelling patients with MS. Generally speaking, the total score can prepare a baseline before the intervention.

\section{Ethical Considerations}

\section{Compliance with ethical guidelines}

The ethical approval was attained from the Ethical Center of Shahid Beheshti University of Medical Sciences.

\section{Funding}

This study was supported by Shahid Beheshti University of Medical Sciences.

\section{Authors contributions}

Conceptualization, methodology, and resources: Meysam Roostaei, Hossein Soltanpour, and Minoo Kalantari; Investigation: Meysam Roostaei and Hossein Soltanpour; Writing original draft, review, and editing: Meysam Roostaei and Minoo Kalantari; Funding acquisition: Hossein Soltanpour, and Minoo Kalantari; Supervision: Minoo Kalantari.

\section{Conflict of interest}

The authors declared no conflict of interests.

\section{References}

[1] Minden S, Frankel D, Hadden L, Perloff J, Srinath K, Hoaglin D. The Sonya Slifka longitudinal multiple sclerosis study: Methods and sample characteristics. Multiple Sclerosis Journal. 2006; 12(1):24-38. [DOI:10.1191/135248506ms1262oa] [PMID]

[2] Pendleton HM, Schultz-Krohn W. Pedretti's occupational therapy-e-book: Practice skills for physical dysfunction. Gurgaon: Elsevier Health Sciences; 2017.

[3] Fritz NE, Marasigan RER, Calabresi PA, Newsome SD, Zackowski KM. The impact of dynamic balance measures on walking performance in multiple sclerosis. Neurorehabilitation and Neural Repair. 2015; 29(1):62-9. [DOI:10.1177/1545968314532835] [PMID] [PMCID]

[4] Fjeldstad C, Pardo G, Bemben D, Bemben M. Decreased postural balance in multiple sclerosis patients with low disability. International Journal of Rehabilitation Research. 2011 34(1):53-8. [DOI:10.1097/MRR.0b013e32833d6ccb] [PMID]

[5] Comber L, Galvin R, Coote S. Gait deficits in people with multiple sclerosis: A systematic review and meta-analysis. Gait \& Posture. 2017; 51:25-35. [DOI:10.1016/j.gaitpost.2016.09.026] [PMID]

[6] Mehravar M, Yadollah-Pour N, Tajali S, Shaterzadeh-Yazdi MJ, Majdinasab N. The role of anticipatory postural adjustments and compensatory control of posture in balance con- 
trol of patients with multiple sclerosis. Journal of Mechanics in Medicine and Biology. 2015; 15(05):1550087. [DOI:10.1142/ S0219519415500876]

[7] Sosnoff JJ, Socie MJ, Boes MK, Sandroff BM, Pula JH, Suh $\mathrm{Y}$, et al. Mobility, balance and falls in persons with multiple sclerosis. PLOS One. 2011; 6(11):e28021. [DOI:10.1371/journal.pone.0028021] [PMID] [PMCID]

[8] Kalron A, Achiron A. The relationship between fear of falling to spatiotemporal gait parameters measured by an instrumented treadmill in people with multiple sclerosis. Gait \& Posture. 2014; 39(2):739-44. [DOI:10.1016/j.gaitpost.2013.10.012] [PMID]

[9] Sutliff MH. Contribution of impaired mobility to patient burden in multiple sclerosis. Current Medical Research and Opinion. 2010; 26(1):109-19. [DOI:10.1185/03007990903433528] [PMID]

[10] Prosperini L, Kouleridou A, Petsas N, Leonardi L, Tona F, Pantano $\mathrm{P}$, et al. The relationship between infratentorial lesions, balance deficit and accidental falls in multiple sclerosis. Journal of the Neurological Sciences. 2011; 304(1-2):55-60. [DOI:10.1016/j.jns.2011.02.014] [PMID]

[11] Tona F, De Giglio L, Petsas N, Sbardella E, Prosperini L Upadhyay N, et al. Role of cerebellar dentate functional connectivity in balance deficits in patients with multiple sclerosis. Radiology. 2017; 287(1):267-75. [DOI:10.1148/radiol.2017170311] [PMID]

[12] Hebert J, Corboy J. The association between multiple sclerosis-related fatigue and balance as a function of central sensory integration. Gait \& Posture. 2013; 38(1):37-42. [DOI:10.1016/j. gaitpost.2012.10.015] [PMID]

[13] Lorefice L, Coghe G, Fenu G, Porta M, Pilloni G, Frau J et al. 'Timed up and go' and brain atrophy: A preliminary MRI study to assess functional mobility performance in multiple sclerosis. Journal of Neurology. 2017; 264(11):2201-4. [DOI:10.1007/s00415-017-8612-y] [PMID]

[14] Fling BW, Dutta GG, Schlueter H, Cameron MH, Horak FB. Associations between proprioceptive neural pathway structural connectivity and balance in people with multiple sclerosis. Frontiers in Human Neuroscience. 2014; 8:814. [DOI:10.3389/fnhum.2014.00814] [PMID] [PMCID]

[15] Jones S. Somatosensory impairment and balance dysfunction in multiple sclerosis. Massachusetts: University of Massachusetts Medical School; 2014.

[16] Shumway-Cook A, Baldwin M, Polissar NL, Gruber W. Predicting the probability for falls in community-dwelling older adults. Physical Therapy. 1997; 77(8):812-9. [DOI:10.1093/ ptj/77.8.812] [PMID]

[17] Shumway-Cook A, Woollacott MH. Motor control: Translating research into clinical practice. Sydney: Lippincott Williams \& Wilkins; 1995.

[18] Jonsdottir J, Cattaneo D. Reliability and validity of the dynamic gait index in persons with chronic stroke. Archives of Physical Medicine and Rehabilitation. 2007; 88(11):1410-5. [DOI:10.1016/j.apmr.2007.08.109] [PMID]

[19] Whitney S, Wrisley D, Furman J. Concurrent validity of the berg balance scale and the dynamic gait index in people with vestibular dysfunction. Physiotherapy Research International. 2003; 8(4):178-86. [DOI:10.1002/pri.288] [PMID]
[20] Simon T, Harro C. Reliability and validity of the dy namic gait index in individuals with brain injury. Journal of Neurologic Physical Therapy. 2004; 28(4):180-1. [DOI:10.1097/01253086-200412000-00038]

[21] McConvey J, Bennett SE. Reliability of the dynamic gait index in individuals with multiple sclerosis. Archives of Physical Medicine and Rehabilitation. 2005; 86(1):130-3. [DOI:10.1016/j.apmr.2003.11.033] [PMID]

[22] Forsberg A, Andreasson M, Nilsagård YE. Validity of the dynamic gait index in people with multiple sclerosis. Physical Therapy. 2013; 93(10):1369-76. [DOI:10.2522/ptj.20120284] [PMID]

[23] Abdiani M, Golpayegani M, Khajavi D. [Validity and reliability of Persian version of dynamic gait index in older men (Persian)]. Iranian Journal of Ageing. 2014; 9(2):124-33.

[24] Eghlidi J, Kalantari M, Soltanpour H, Akbar-Fahimi M, Akbarzadeh A, Beladi MN, et al. [Validity of the Persian version of Dynamic Gait Index (DGI-P) in individuals with multiple sclerosis (Persian)]. Journal of Rehabilitation Medicine. 2017; 6(2):11-8.

[25] Kottner J, Audigé L, Brorson S, Donner A, Gajewski BJ, Hróbjartsson A, et al. Guidelines for Reporting Reliability And Agreement Studies (GRRAS) were proposed. International Journal of Nursing Studies. 2011; 48(6):661-71. [DOI:10.1016/j.ijnurstu.2011.01.017] [PMID]

[26] Herman T, Inbar-Borovsky N, Brozgol M, Giladi N, Hausdorff JM. The dynamic gait index in healthy older adults: The role of stair climbing, fear of falling and gender. Gait \& Posture. 2009; 29(2):237-41. [DOI:10.1016/j.gaitpost.2008.08.013] [PMID] [PMCID]

[27] Andresen EM. Criteria for assessing the tools of disability outcomes research. Archives of Physical Medicine and Rehabilitation. 2000; 81(Suppl. 2):S15-20. [DOI:10.1053/ apmr.2000.20619]

[28] Cattaneo D, Jonsdottir J, Repetti S. Reliability of four scales on balance disorders in persons with multiple sclerosis. Disability and Rehabilitation. 2007; 29(24):1920-5. [DOI:10.1080/09638280701191859] [PMID]

[29] Socie MJ, Sosnoff JJ. Gait variability and multiple sclerosis. Multiple Sclerosis International. 2013; 2013:645197. [DOI:10.1155/2013/645197] [PMID] [PMCID]

[30] Goldsmith JW. Methodological considerations in using videotape to establish rater reliability. Nursing Research. 1981; 30(2):124-8. [DOI:10.1097/00006199-198103000-00016] [PMID]

[31] Soyuer F, Mirza M, Erkorkmaz Ü. Balance performance in three forms of multiple sclerosis. Neurological Research 2006; 28(5):555-62. [DOI:10.1179/016164105X49373] [PMID] 
This Page Intentionally Left Blank 